BUONE PRASSI- BEST PRACTICES

\author{
LA CASA COME SPAZIO EDUCANTE: \\ RIFLESSIONI PEDAGOGICHE A PARTIRE DA UNO \\ STUDIO DURANTE IL LOCKDOWN
}

\title{
THE HOUSE AS AN EDUCATING SPACE. PEDAGOGI- CAL REFLECTIONS STARTING FROM A STUDY DURING THE LOCKDOWN
}

\section{di Elisabetta Biffi e Lucia Carriera (Università degli Studi di Milano Bicocca)*}

Il presente contributo si propone di esplorare e riflettere, da una prospettiva pedagogica, sulla casa come luogo di educazione per le bambine e i bambini che la abitano attraverso la prospettiva offerta dall'emergenza pandemica in corso. Nella prima infanzia la dimora della famiglia rappresenta il luogo entro il quale il bambino comincia a prendere coscienza di sé, e a esistere proprio a partire dallo spazio della casa. L'emergenza COVID-19 ha imposto un ritorno forzato alle mura domestiche portando a ripensare il ruolo della casa. Si intende, pertanto, condividere alcune riflessioni pedagogiche a partire dai risultati di una ricerca qualitativa (parte del progetto Europeo Erasmus+ DEPCIP) condotta durante il lockdown di marzo-maggio 2020 e volta a comprendere l'impatto che un periodo prolungato di confinamento ha avuto sull'ambiente familiare. Il contributo si basa su una ricerca quali-quantitativa (Teddlie \& Tasshakori, 2006), condotta attraverso l'intervista semistrutturata Computer Assisted Web Interview (CAWI). Circa 1000 genitori provenienti dai Paesi coinvolti nel progetto sono stati raggiunti, di cui 400 italiani. Lo studio ha inoltre indagato le relazioni con gli spazi domestici e pubblici, mostrando come l'isolamento

* Il seguente contributo è frutto di un lavoro congiunto delle Autrici. Nello specifico, sono da considerarsi opera di Elisabetta Biffi i paragrafi 3 e 4; sono da considerarsi opera di Lucia Carriera i paragrafi 1 e 2. 
abbia provocato una ridefinizione delle "geografie di casa" delle famiglie (Blunt \& Varley, 2004). A partire dai risultati di ricerca il presente paper rifletterà sulla complessità dei significati di casa che si sono andati attraversando nel corso di questo periodo emergenziale e delle sue ricadute sul piano educativo.

This contribution aims to explore and reflect, from a pedagogical perspective, on the home as a place of education for the children who live there, from the perspective offered by the current pandemic emergency. In early childhood, the family home is the place where children begin to become aware of themselves (Leccardi et al., 2011), and to exist precisely from the space of the home (Giordano, 1997). The COVID-19 emergency has forced a return to the home, leading us to rethink the role of the home.

We therefore intend to share some pedagogical reflections based on the results of a qualitative research (part of the European project Erasmus + DEPCIP) conducted during the lockdown of March-May 2020 and aimed at understanding the impact that a prolonged period of confinement has had on the family environment. The contribution is based on qualitative-quantitative research (Teddlie \& Tasshakori, 2006), conducted through the semistructured Computer Assisted Web Interview (CAWI). The study reached, through written interviews, about 1000 parents in lockdown from the countries involved in the project, of which about 400 Italian parents. The study also investigated the relationships with domestic and public spaces, showing how isolation has caused a redefinition of the families' "home geographies" (Blunt \& Varley, 2004). Starting from the results of the research, this paper will reflect on the complexity of the meanings of home that have been experienced during this emergency period and its repercussions on the educational level. 


\section{La casa come spario educante}

L'idea di casa è un concetto complesso, multidimensionale e pluristratificato, nel suo essere un luogo fisico, un set di significati e la relazione tra questi due (Blunt \& Dowling, 2006, p. 2). Riprendendo una definizione offerta da Shelley Mallett (2004), la casa si configura come luogo virtuale, deposito di memorie e di spazi vissuti (p. 63) dove poter localizzare il tempo e lo spazio familiare. Spesso, in letteratura, la parola casa viene associata alla dimensione del nido, della sicurezza, o più romanticamente a quella del focolare. Essa rimanda spesso alla dimensione familiare, tanto da essere usata come sinonimo stesso della parola famiglia (Crow, 1989; Oakley, 1976). Tuttavia, per quanto rimandi a una dimensione intima e privata, l'associazione casa-sicurezza è qualcosa che di per sé non può affatto essere dato per scontato (Biffi, 2020).

La casa è, infatti, uno dei contesti nei quali è possibile osservare forse più accuratamente $\mathrm{i}$ «guai» di una famiglia (Evans, Bowlby, Gottzén \& Ribbens McCarthy, 2019) e le complesse relazioni intragenerazionali, le solitudini, le vulnerabilità dei suoi abitanti.

Parlando della relazione tra casa e famiglia, particolarmente rilevante è la riflessione a opera di Vanna Iori (2008) che, nel testo Lo spazio vissuto, enfatizza quanto il senso di casa rappresenti un'altra faccia del sentimento familiare (p. 175). Infatti, le stesse trasformazioni della casa nella storia, come ricorda l'autrice, hanno implicato una parallela trasformazione della famiglia.

Per tale ragione, secondo Chombart de Lawe la relazione tra abitazione e struttura familiare è così forte da poter parlare di una corrispondenza tra "unità spaziale e unità sociale" (Ceci, 1996).

Si pensi per esempio al Medioevo, in cui il bambino di sette anni circa viveva in prossimità con gli adulti, lavorando e inserendosi in una vita collettiva e priva di intimità (Iori, 2008, p. 174), senza ricevere particolare educazione in preparazione alla vita adulta. Un altro esempio è altresì offerto dall'ambiente urbano dell'Europa tra il Sei-Settecento con la diffusione, tra i lavoratori poveri dei grandi centri urbani, del lavoro a domicilio. Gli artigiani, 
non disponendo di capitale per poter aprire una bottega, impiantarono i propri laboratori nelle abitazioni stesse. Spesso vi era un'unica stanza, adibita a laboratorio e cucina, e i letti erano stipati in un soppalco (Pancera, 2001, p. 126). Data la ristrettezza degli ambienti, i membri della famiglia vivevano in una prossimità promiscua, totalmente priva di intimità.

Questi sono solo alcuni esempi offerti dalla storia per illustrare, senza alcuna pretesa esaustiva, come le trasformazioni e gli utilizzi della casa abbiano implicato una parallela trasformazione delle relazioni familiari e del significato dell'infanzia e abbiano condotto a un'evoluzione anche della pedagogia dell'abitare domestico (Iori, 2008, p. 174).

Non sono soltanto le connessioni fra la casa e la dimensione familiare a rendere tale oggetto di particolare interesse per la riflessione pedagogica. Nella prima infanzia la casa rappresenta, infatti, il luogo entro il quale il bambino comincia a prendere coscienza di sé (Leccardi et al., 2011) e a esistere proprio a partire dallo spazio della casa (Giordano, 1997). Tale aspetto si ricollega alla riflessione di Bachelard (1993), per il quale la casa è il nostro primo universo e tutto lo spazio realmente abitato porta con sé la nozione di casa. Heller, in un saggio pubblicato nel 1994 intitolato Dove ci sentiamo a casa?, sottolinea la capacità dello spazio fisico nel proporsi come testimone e al tempo stesso custode della memoria culturale. Possiamo, secondo tale visione, sentirci a casa in un luogo dove sono maturate delle tradizioni culturali che lo rendono tale. Un luogo, dunque, in cui gli abitanti possano condividere uno stesso spazio discorsivo in cui la tradizione prende forma nella prassi quotidiana, una prassi intessuta di comunicazioni che rimandano a un retroterra cognitivo già presupposto (Heller, 1994; Leccardi et al., 2011), uno spazio che potremmo anche definire dell'abitudine.

L'intimità accogliente della casa si fonda sul contatto visivo, uditivo, olfattivo e sui vissuti abituali. Abitare, abitudine, abituale hanno infatti la stessa origine del verbo habitare, frequentativo di habere che significa "avere" nel senso di occupare, padroneggiare, possedere, dominare. Noi infatti padroneggiamo attraverso le abitudini percettive lo spazio abitativo (Iori, 2008, p. 177). 
La casa è, inoltre, anche un luogo intenzionalmente educativo, sia quando pensata nella sua organizzazione dagli adulti per i bambini e le bambine che la abitano, sia quando sede di interventi educativi specifici, come accade ad esempio con gli interventi di assistenza domiciliare rivolti ai minorenni (Panattoni, 2017). «Un servizio di educativa domiciliare, infatti, ha nella casa il luogo in cui progettualmente deve avvenire l'interazione educativa» (Premoli, Confalonieri \& Volpi, 2012, p. 93). Interazione che dunque avviene in un setting pre-esistente, denso di memorie, di pratiche e di significati. In tal senso, questo oggetto si apre come spazio di vita che si fa tramite, diretto e indiretto, di un progetto educante per i soggetti che vi abitano.

\section{La dialettica interno/esterno: l'impatto della pandemia}

A fronte di queste prime riflessioni, è possibile ora interrogarsi sulla dialettica tra interno ed esterno che circonda la casa.

Un osservatorio privilegiato è stato quello offerto dallo scenario che si è andato tracciando nel corso della prima fase di emergenza dovuta alla pandemia da COVID-19, agli inizi del 2020. La pandemia ha, infatti, impattato profondamente sulla quotidianità e la vita di ciascun individuo imponendo per molti mesi, durante i momenti di lockdown, un ritorno forzato alle mura domestiche. Le restrizioni attuate per attenuare il rischio di contagio hanno inevitabilmente limitato la possibilità per bambine e bambini di frequentare molti dei luoghi abitualmente vissuti all'esterno della propria abitazione. Primi fra tutti la scuola e i servizi alla prima infanzia, ma anche i parchi gioco e i luoghi di aggregazione in generale. Tale condizione eccezionale ha condotto a un vero e proprio stravolgimento delle soglie: la convivenza obbligata dell'intero nucleo familiare all'interno della stessa abitazione ha, infatti, costretto a ripensare gli spazi, per consentire le diverse attività, dalla scuola al lavoro, ponendo anche una questione non irrilevante di privacy, di 
fronte all'imperversare delle videocamere accese dei diversi dispositivi elettronici, per la didattica a distanza, lo smartworking, le comunicazioni con familiari e amici.

In questo senso, la pandemia e l'esperienza di lockdown attraversata hanno posto l'attenzione sulla dialettica - più che sulla dicotomia - tra interno ed esterno e sulle soglie dell'abitare la casa. Tali dimensioni rappresentano, infatti, dei tratti intrinsecamente costitutivi dell'abitare la casa, necessari allo sviluppo armonico della formazione umana. Interrogare la casa e le modalità tramite le quali le famiglie hanno vissuto gli spazi domestici durante il lockdown consente, così, di raggiungere un osservatorio inedito che apre a possibili piste di sviluppo della riflessione pedagogica.

\section{Riflessioni da uno studio}

All'interno di questa cornice, il presente articolo vuole offrire l'osservatorio raggiunto attraverso uno studio esplorativo che ha interrogato le famiglie durante il primo periodo di lockdown in Italia, tra marzo e giugno 2020.

Tale studio è parte di un più vasto progetto europeo Erasmus+ Digitised Education of Parents for Children Protection (DEPCIP) ${ }^{1}$, il cui obiettivo è sviluppare proposte formative, anche attraverso specifici strumenti digitali, dedicate ai genitori per la promozione dei diritti dei minorenni e la riduzione del rischio di violenza sull'infanzia. Durante il corso del 2020 il progetto ha realizzato uno studio esplorativo volto a comprendere l'impatto che un periodo prolungato di confinamento stava avendo sull'ambiente familiare. Si è trattato di una ricerca quali-quantitativa (Teddlie \& Tasshakori, 2006), condotta attraverso l'intervista semi-strutturata Computer Assisted Web Interview $(\mathrm{CAWI})^{2}$. L'indagine ha raggiunto circa un

1 Per maggiori approfondimenti si rimanda a: http://depcip.com/ [10/11/2021].

${ }^{2} \mathrm{Si}$ è trattato di un disegno di ricerca esplorativo a matrice mista (Teddlie \& Tashakkori, 2006). La struttura dell'intervista era così articolata: informazioni 
migliaio di "genitori in lockdown" provenienti dai Paesi coinvolti nel progetto (nello specifico Grecia, Lituania, Italia, Spagna e Turchia), attraverso interviste orali e scritte. In Italia la ricerca ha coinvolto poco meno di 400 genitori, di cui più del $90 \%$ madri residenti in Lombardia, una delle regioni più colpite dalla pandemia ${ }^{3}$.

La ricerca si è focalizzata sull'indagine dell'ecosistema familiare, esplorando dimensioni individuali (stati emotivi, disponibilità di risorse, supporto ricevuto, benessere e vulnerabilità), aspetti spaziali (trasformazione dei luoghi, attribuzione di nuovi significati) e aspetti genitoriali nel rapporto con i figli (la comunicazione dell'emergenza sanitaria, la gestione di situazioni difficili e di criticità).

Dentro al più complesso quadro di ricerca, che qui non è possibile riportare per esteso ${ }^{4}$, ai fini della riflessione in corso ci si soffermerà sulla parte di indagine dedicata, appunto, alla riflessione sullo spazio. Lo studio ha, infatti, indagato le relazioni con gli spazi domestici e pubblici, mostrando come l'isolamento abbia provocato una ridefinizione delle "geografie di casa" delle famiglie (Blunt \& Varley, 2004). In generale, gli intervistati hanno raccontato della fatica di dover riorganizzare la propria routine in tempi rapidi e facendo fronte a circostanze del tutto nuove, come ad esempio il lavoro da casa e la didattica a distanza, e delle difficoltà che la ricerca di nuovi equilibri nelle dinamiche con i figli ha comportato.

Per ciò che concerne gli spazi, di particolare rilevanza è stata l'attenzione al cambiamento nel modo di vivere gli spazi, non solo privati, ma anche comuni, limitrofi appunto alla soglia di casa. Di fronte alla richiesta di pensare alla propria relazione con gli spazi

socio-demografiche; routine quotidiane e gestione degli spazi; genitorialità in tempo di lockdown; dimensioni emotive e psicologiche; domande aperte, scale Likert, misure standardizzate.

${ }^{3}$ In riferimento al campione, si segnala che si è trattato per la maggioranza di madri $(93 \%)$ di famiglie bi-genitoriali (80\%), con età compresa tra 29 e 57 anni $(\mathrm{M}=41.9, \mathrm{SD}=6.04)$. Il numero medio di figli $=1.9$, con età media di circa 8 anni (range 6 mesi - 18 anni) e un livello educativo molto alto (57\% grado universitario o superiore).

${ }^{4}$ Per approfondimenti: Biffi, Gambacorti-Passerini \& Bianchi, 2021. 
comuni (giardino, pianerottolo, androni, corselli dei box) ${ }^{5}$, un significativo $18 \%$ ha indicato il lockdown come opportunità per ripensare la propria relazione con questi spazi. È emersa la volontà di provare a viverli maggiormente e in modo nuovo, per dare loro nuovi significati (molti dei quali collegati all'utilizzo di tali spazi con i bambini). È quanto ben testimoniano da risposte come le seguenti: «Ho sistemato il balcone e non lo facevo così da 10 anni e ora possiamo mangiare fuori. Abbiamo guadagnato un luogo in più». «Certamente li viviamo di più, aumentando, paradossalmente, le interazioni con gli altri [...] questo ci ha permesso anche di supportarci a vicenda e vivere più serenamente questa situazione».

Per quanto riguarda i luoghi che sono mancati di più durante il lockdown ${ }^{6}$, le risposte raccolte lasciano emergere l'idea dello spazio pubblico come relazione: la maggior parte dei rispondenti ha fatto riferimento non tanto a luoghi ma ad azioni e modi di vivere lo spazio insieme agli altri, tanto per cui a mancare sono i parchi $(40 \%)$, le biblioteche (14\%), i luoghi dove ci si incontra.

Tali considerazioni portano a indicare quanto la casa sia da pensarsi nella relazione con ciò che le è intorno, come spazio che assume senso anche nella relazione con il fuori, in una ridefinizione del concetto di intimità particolarmente fruttuoso per la riflessione pedagogica (Biffi, 2020). L'essere umano e il bambino in particolar modo, devono poter vivere nel mondo. «L'esperienza dell'abitare non può avvenire solo percorrendo pavimenti e collocando il proprio corpo all'interno di edifici chiusi» (Amadini, 2016, p. 96). Privare i bambini di tale esperienza comporta una profonda trasformazione del rapporto tra corpo e spazio, ed è proprio ciò che è avvenuto in seguito alle restrizioni vissute nei mesi di lockdown. «La città è un linguaggio [...]. Proprio come una lingua delimita ciò che può essere detto, così l'architettura traccia i limiti entro i quali si

${ }^{5}$ Domanda per esteso: "Se vive in un condominio come è cambiata, se è cambiata, la sua relazione con gli spazi comuni (giardino, pianerottolo, androni, corselli dei garage)?”.

${ }^{6}$ Domanda per esteso: "Se pensa al quartiere in cui risiede (o al piccolo centro) quali sono i luoghi che frequentava con i suoi figli che più le mancano e di cui avverte maggiormente l'assenza?". 
può camminare, ma chi cammina inventa altri modi di muoversi» (Solnit, 2002, p. 243). In questa riflessione di Rebecca Solnit (2002), contenuta all'interno del testo Storia del camminare, si può rintracciare quella stessa tendenza messa in atto all'interno delle case di cui si è parlato nelle righe precedenti. Ecco come attraverso la riconfigurazione e la risignificazione di alcuni spazi, si sono rintracciate nuove traiettorie dell'abitare. Emerge dagli intervistati il tentativo di generare connessioni inedite con l'esterno, attraverso la ricostruzione di certi spazi - si pensi ad esempio ai balconi - inventando, nonostante i vincoli "architettonici" imposti dalla pandemia, nuovi modi per muoversi.

\section{Conclusioni}

Come ben evidenzia Monica Amadini, riprendendo la riflessione di Gaston Bachelard (1975), la casa è uno stato d'animo, anche riprodotto dal suo aspetto esterno, e rivela un'intimità. Vivere il dentro della propria casa fa sentire all'individuo di avere un posto nel mondo:

[i]l bambino disegna la casa nella sua forma, ma quasi sempre qualche tratto designa un'intima forza [...]. Nel disegno della casa i bambini rivelano i sogni più profondi, il riparo della loro felicità, le radici, il calore, come pure la casa può recare la traccia delle ansie del disegnatore (Bachelard, 1975, cit. in Amadini, 2016, p. 63).

La casa si configura, come già ribadito, quale luogo d' intimità, tanto che per poter varcare la sua soglia - fisica, ma soprattutto psicologica - siamo soliti chiedere permesso a chi la abita (Iori, 2008, p. 170).

Questa stessa dimensione di intimità è rintracciabile in quegli spazi che, all'interno della casa, parlano di un dentro ancora più dentro. La casa e la stanza non sempre costituiscono rifugi sufficientemente sicuri per i loro abitanti (Iori, 2008, p. 183). Talvolta i bambini sono alla ricerca di uno spazio ancora più interno. Si pensi, per esempio, alla creazione dell'angolo segreto, sotto forma di fortino, 
rifugio o piccola tana, edificato tra i mobili della casa, sotto le scalinate o negli angoli più disparati grazie a coperte, cuscini e lenzuola.

Un altro esempio è offerto dagli spazi personali come la camera da letto e/o da gioco per gli adolescenti, quel luogo che cresce insieme ai ragazzi e al quale fare ritorno dopo aver sperimentato il mondo esterno (Iori, 2008, p. 181). Come ricorda Amadini (2016), sono esperienze dal profondo valore simbolico e corrispondono a un bisogno di protezione e raccoglimento (p. 92). L'interno, continua l'autrice, costituisce dunque il nostro angolo nel mondo, e se nell'educazione tale luogo fisico e interiore viene a mancare, il soggetto non ha dove stare.

Tuttavia, posta la dimensione profondamente intima della casa, le riflessioni a seguito del periodo di confinamento hanno rivelato quanto essa non debba essere pensata quale luogo di separazione dal resto del mondo, un guscio o una barriera, e sul piano della riflessione pedagogica rappresenta un oggetto da tematizzare. Soprattutto in considerazione del fatto che la casa stessa non è luogo necessariamente sicuro per chi la abita, come dimostrano le numerose ricerche sulla violenza domestica, appunto, verso minorenni e donne, in prevalenza.

Si è, così, forse oggi chiamati a una riflessione sulla casa che consenta di ripensarla in una nuova relazione fra privato e collettivo. Questo duplice volto interno/esterno illustra la duplice valenza, privata e pubblica, della casa: tali vie dell'abitare sono dimensioni costitutive dell'esistenza umana, e possono essere associate alle figure mitologiche di Hestia ed Hermes (Vernant, 1970, cit. in Iori, 2008, p. 168). Hestia rappresenta nel mito greco il centro simbolico dello spazio domestico. È intimità e immobilità, la fissità attraverso cui si organizza lo spazio umano. Hermes rappresenta lo spazio complementare ad Hestia: è lo spazio cinetico, la transizione, il passaggio. Anch'egli è nella casa, ma rimane sulla soglia. Lo spazio, dunque, esige un centro dotato di un valore privilegiato a partire dal quale poter orientare le direzioni umane, a partire dal quale prendere spazio nel mondo. L'intimità della casa in questo senso, ricorda Amadini (2016), è la condizione per aprirsi al mondo 
e situarsi in esso. Evocativa è in tal senso l'immagine di Ulisse: potrà viaggiare per molti anni, lontano dalla propria patria, solcando i mari alla ricerca di nuove terre, perché nella sua mente esisterà sempre una piccola Itaca a cui poter fare ritorno, un punto fermo nello spazio dal quale poter tornare.

Tornando alla nostra contemporaneità, la situazione pandemica, attualmente ancora in atto, ha condotto ancor più al ripensamento di tale dialettica. Ha esacerbato fragilità esistenti, celandole e - in certi casi - persino soffocandole fra le trame dell'“intimità" domestica. Ha amplificato spazi di separazione generando isolamento: si pensi per esempio alle bambine e ai bambini accolti nelle comunità educative o agli anziani accolti nelle RSA. Ha portato da un lato alla risignificazione degli spazi del quotidiano e a quegli spazi liminali di passaggio. Ha posto in luce quanto la casa sia un luogo poroso, in cui le relazioni personali che essa ospita attraversano il mondo pubblico e quello politico (Brickell, 2012).

Incrinando in questo senso l'idea di dimora come micro-cosmo sviluppato unicamente verso l'interno. Ha mostrato la presenza talvolta prevaricante - di "Hermes" in "Hestia" e viceversa, generando nuovi apprendimenti, nuovi spunti per la riflessione pedagogica.

\section{Bibliografia}

Amadini M. (2016). I bambini e il senso dell'abitare. Prospettive di ricerca pedagogica. Parma: Junior-Spaggiari.

Bachelard G. (1993). La poetica dello spazio (Vol. 30). Bari: Dedalo. Biffi E. (2020). Il rovescio della trama educativa. Milano: FrancoAngeli.

Biffi E., Gambacorti-Passerini M.B., \& Bianchi D. (2021). Parents under Lockdown: the Impacts of the COVID-19 Pandemic on Families. Rivista Italiana Di Educazione Familiare, 18(1), 97-111.

Blunt A., \& Dowling R. (2006). Home. London: Routledge.

Blunt A., \& Varley A. (2004). Geographies of home. Cultural geographies, 11, 3-6. Brickell K. (2012). "Mapping" and "doing" critical geographies of home.

Progress in human geography, 36(2), 225-244.

Ceci L.R. (1996). La città, la casa, il valore: borghesia e modello di vita urbano. Roma: Armando. 
Coppola Pignatelli P. (1982). Spario e immaginario. Roma: Officina Edizioni.

Crow G. (1989). The post-war development of the modern domestic ideal. In G. Allan \& G. Crow (eds.), Home and family: creating the domestic sphere (pp. 14-32). London: Palgrave Macmillan.

Evans R., Bowlby S., Gottzén L., \& Ribbens McCarthy J. (2019). Unpacking "family troubles", care and relationality across time and space. Children's Geographies, 17(5), 501-513.

Heidegger M. (1976). Saggi e discorsi. Milano: Mursia.

Heller A. (1994). Dove ci sentiamo a casa? il Mulino, 43(3), 381-399.

Iori V. (2008). Lo spazio vissuto: luoghi educativi e soggettività. Milano: FrancoAngeli.

Mallett S. (2004). Understanding home: a critical review of the literature. The sociological review, 52(1), 62-89.

Oakley A. (1974). The Sociology of Housework. New York: Random House Inc. Oakley A. (1979). Becoming a Mother. New York: Schocken Books.

Panattoni C. (2017). L'assistenza domiciliare educativa rivolta a minori e famiglie. Un intervento psicoanaliticamente orientato a partire dal ruolo di educatore. Quaderni della Rivista di Psicologia Clinica, 1, 24-34.

Pancera C. (2001). La socializzazione dell'infanzia lavoratrice nell'età preindustriale. In C. Covato \& S. Ulivieri (a cura di), Itinerari nella storia dellinfanzia: bambine e bambini, modelli pedagogici e stili educativi (pp. 125146). Milano: Unicopli.

Premoli S., Confalonieri M., \& Volpi M. (2012). In terra straniera serve tempo. Entrare come educatrici a casa di bambini e genitori vulnerabili. Animazione sociale, 43(259), 91-101.

Solnit R. (2002). Storia del camminare. Milano: B. Mondadori.

Teddlie C., \& Tashakkori A. (2006). A general typology of research designs featuring mixed methods. Research in the Schools, 13(1), 12-28.

Vernant P. (1970). Mito e pensiero presso i Greci. Torino: Einaudi. 